\title{
Oberarmschaftfrakturen - Marknagelosteosynthese
}

\author{
Jochen Blum, Erik Hanke, Philip Höhle, Pol M. Rommens
}

\section{Zusammenfassung}

Neben der konservativen Behandlung wurde zur operativen Behandlung der Oberarmschaftfrakturen lange Zeit die Plattenosteosynthese propagiert. Vielfältige intramedulläre Verfahren standen zwar zur Verfügung, konnten jeweils aber keine sehr große Verbreitung finden. Inzwischen lässt sich hingegen ein deutlicher Trend zur Verriegelungsmarknagelung am Oberarm erkennen. Dies hängt primär mit einem verfeinerten Design dieser Marknägel und deren Instrumentarium, aber auch verbesserten OP-Techniken zusammen. Die Erfahrung mit dem unaufgebohrten Humerusverriegelungsnagel (UHN) für reine Schaftfrakturen, dem UHN mit winkelstabiler Spiralklingenverriegelung für die Kombination ipsilateraler dia- und metaphysären Frakturen, wie auch mit dem proximalen Humerusnagel (PHN) für sehr proximal gelegene Schaftfrakturen bestätigen den Sinn dieses Trends. Dies betrifft einerseits das intraoperative Handling, aber auch die postoperativen Ergebnisse hinsichtlich Frakturheilung und Bewegungsfunktion. Neben den akuten Monofrakturen umfasst das Indikationsspektrum auch die Humerusfraktur bei mehrfachverletzten oder polytraumatisierten Patienten, aber auch Refrakturen, Pseudarthrosen und pathologische Frakturen. Wie bei anderen Implantatsystemen auch, hängen Erfolg und Misserfolg mit diesem Nagelsystem für Oberarmschaftfrakturen eng mit der Beachtung grundlegender implantationstechnischer Details ab. Dies betrifft vor allem die Morbidität der Zugangswege, das iatrogene Risiko von Nerven- und Gefäßverletzungen und zusätzlicher Fissuren oder gar Frakturen, aber auch die möglichst ungestörte und infektfreie Knochenheilung. Eine Indikation zur operativen Versorgung vorausgesetzt, kann der UHN/PHN als sicheres und ausgereiftes Implantat zum Einsatz bei Humerusschaftfrakturen empfohlen werden.

\section{Einleitung}

Oberarmschaftfrakturen können akut als isolierte Fraktur, im Rahmen von Mehrfachverletzungen oder bei Polytraumen auftreten, hier meist im Rahmen eines direkten Traumas. In $2 / 3$ der Fälle handelt es sich um niederenergetische Verletzungsmechanismen. Insgesamt stellen sie etwa $1 \%$ aller Frakturen dar, davon sind wiederum ca. $6 \%$ offen. Als pathologische Frakturen sehen wir sie infolge benigner oder maligner Erkrankungen, allerdings in der Mehrzahl durch extra-ossäre Metastasierung. Hier stellt das Mam-

OP-JOURNAL 2002; 18: 252-261

(c) Georg Thieme Verlag Stuttgart . New York makarzinom den häufigsten ursächlichen Tumor dar.

Generell kontrovers wird das geeignete therapeutische Verfahren diskutiert. Die meisten Vorträge zu dieser Thematik beginnen mit der Einleitung, dass die Humerusschaftfraktur eine Domäne der konservativen Behandlung sei, die klinische Realität zeigt allerdings gerade in den letzten Jahren eine deutliche Zunahme in der Indikationsstellung zum operativen Verfahren.

Zweifellos können diese Frakturen in vielen Fällen durch konservative Verfahren stabil zur Ausheilung gebracht werden [7]. Dies hängt mit der prinzipiell günstigen Heilungstendenz aufgrund guter
Blutversorgung, wie auch damit zusammen, dass Rotations- und Achsenfehlstellungen unter $20^{\circ}$ funktionell gut kompensiert und kosmetisch gut akzeptiert werden. Allerdings sind daraus resultierende Forderungen nach einem generell konservativen Verfahren als überzogen einzustufen.

Unzufriedenheit gegenüber dem konservativen Vorgehen in speziellen Fraktursituationen des Humerusschaftes bildet die Grundlage, seit mehreren Jahrzehnten nach geeigneten operativen Verfahren zu fahnden, die in diesen Fällen eine stabile Versorgung, aber auch rasche Rehabilitation gewährleisten können.

Als empfohlene OP-Indikationen bei Humerusschaftfrakturen sind die Querfraktur, die gleichseitige Ober- und Unterarmfraktur, beidseitige Schaftfrakturen, die nicht akzeptable Stellung nach geschlossener Reposition, wie auch die begleitende Lähmung des Nervus radialis anzusehen. Eine absolute Indikation besteht bei offenen Oberarmschaftfrakturen, Frakturen mit Gefäßverletzung, beim polytraumatisierten Patienten und bei der Pseudarthrose.

Traten endomedulläre Verfahren, wie die Versorgung mittels Ender- und Hackethal-Nägeln oder Rush-Pins aus Stabilitätsgründen im Vergleich zu der lange Jahre von der AO favorisierten Plattenosteosynthese eher in den Hintergrund $[1,8,11,22]$, scheinen stabile neue Humerusmarknägel in der Lage zu sein, den Plattennachteil der relativ hohen Rate an Schädigungen des N. radialis zu senken [15] und dennoch die gewünschte Stabilität zu gewährleisten. Die Verwendung intramedullärer Nagelsysteme zur Versorgung von Schaftbrüchen wird bei Femur und Tibia vielerorts nicht mehr nur als Alternative zur Plattenosteosynthese gehandelt, sondern je nach Frakturtyp und -lokalisation durchaus als Standardverfahren angesehen. So ist im Falle der Humerusschaftfraktur der Wunsch verständlich, diese ebenfalls durch einen 
intramedullären Nagel versorgen zu können.

Der Humerus unterscheidet sich von diesen beiden anderen langen Röhrenknochen durch unterschiedliche klinische und biomechanische Hintergründe. Anatomische Besonderheiten des Humerus, aber auch seine von Femur und Tibia unterschiedliche Beanspruchung seitens Zug-, Druck-, Biege- und insbesondere Rotationskräfte erfordern eine spezifische Betrachtung dieses durch Muskelkraft und nicht durch Körpergewicht belasteten langen Röhrenknochens im Blickwinkel einer bevorstehenden Frakturversorgung. Gefragt sind Implantatsysteme, die insbesondere den Torsionskräften ausreichenden Widerstand bieten können, was für die Verriegelungsmarknagelung zutrifft. Dies macht sich besonders bei den Quer- und kurzen Schrägfrakturen des Humerusschaftes bemerkbar, welche aufgrund geringer Fragementverzahnung torsionsinstabil sind und zur Pseudarthrosenbildung neigen. Hier ist sogar eine zusätzliche interfragmentäre Kompression wünschenswert, welche mit dem unaufgebohrten Humerusnagel der AO (UHN) realisiert werden konnte $[2,3,4,18,19,20]$. Dieser speziell für den Humerus konzipierte Verriegelungsmarknagel ermöglicht sowohl einen antegraden als auch retrograden Zugangsweg. In der Summe konnte durch diese Konzeptionierung sowohl biomechanisch als auch klinisch eine deutliche Verbesserung gegenüber vorbestehenden Humerusmarknägeln, wie beispielsweise dem Russell-Taylor-Nagel $[9,16,17]$ oder dem Seidel-Nagel $[10,12,13,14,21,23]$ bewirkt werden.

Der antegrade Zugang zum Humerusschaft wird ausgehend vom AkromioKlavikular-Gelenk durch eine kleine anterolaterale Inzision realisiert, der extraartikuläre, retrograde Zugang über eine dorsale Insertionsbohrung am distalen Humerus.

Während letzterer die geringste Gelenkkompromittierung verspricht, lässt sich der antegrade Zugang in Rückenlagerung bzw. Beach-chair-Position durchführen. Es bleibt im Einzelfall abzuwägen, welcher Zugang individuell am günstigsten für den betreffenden Patienten ist [3] Ein zusätzlich komplizierendes Moment ist die Kombination der Oberarmschaftfraktur mit meta- oder epiphysären Frakturanteilen. Im Bereich des proximalen Humerus ermöglicht eine neue Verriegelungsoption des UHN mit einer Spiral-

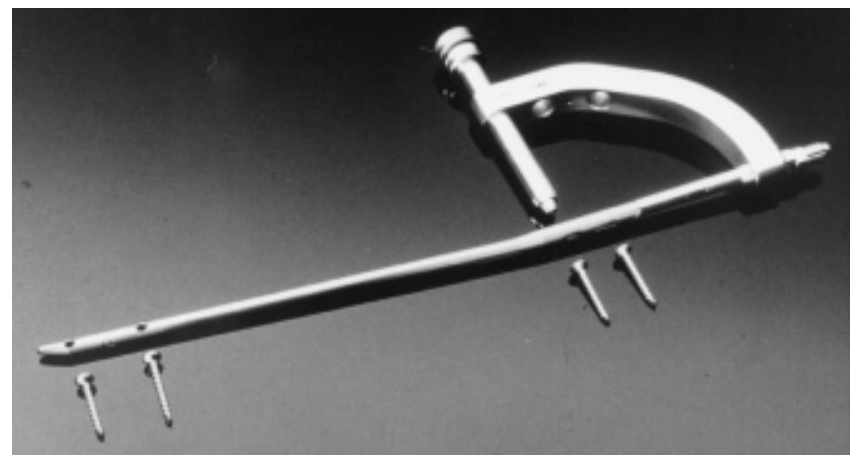

Abb.1 Der unaufgebohrte Humerusnagel (UHN) aus Ti-6Al-7Nb (Mathys AG, CH-Bettlach). Nagelmontage mit Zielbügel. An der Nagelbasis ist das Nagelrohr um $5^{\circ}$ seiner Achse gekrümmt, die Nagelspitze ist abgeschrägt und abgerundet. An der Nagelspitze kann einfach, doppelt oder dreifach durch die hier sichtbaren Verriegelungslöcher verriegelt werden. Die Nagelbasis wird für Schaftfrakturen mit Verriegelungsbolzen aus der gleichen Titanlegierung entweder doppelt parallel oder einfach schräg besetzt.

klinge, auch solche Frakturkombinationen mittels Marknagelung zu versorgen. Dies stellt eine gute biologische Alternative gegenüber der sonstigen Plattenosteosynthese mit langstreckiger Dissektion dar. Eine Kurzversion dieses Nagels, der „proximale Humerusnagel“ (PHN), der ebenfalls mit dieser Spiralklinge kombiniert wird, erleichtert die Verriegelungsnagelung bei sehr proximalen Humerusschaftfrakturen, ist aber speziell für die proximalen Humerusfrakturen der Typen 11-A und 11-B konzipiert [6].

\section{Implantate}

Beide Verriegelungsmarknägel für den Humerus, der UHN (unaufgebohrter Humerusnagel, Synthes ${ }^{\circledR}$, Abb.1) und der PHN (Proximaler Humerusnagel, Synthes $^{\circledR}$, Abb.5) sind aus Titan gefertigt und sowohl proximal als auch distal, verriegelbar.

Hierbei liegt der UHN in drei Durchmessern von 6,7, 7,5 und 9,5 mm, der PHN von 7,5 und $8 \mathrm{~mm}$ vor.

Die Nagelbasis des UHN ermöglicht die Bolzenbesetzung parallel im dynamischen und statischen Verriegelungsloch, oder alternativ in einem nach medial schräg abfallenden Loch. Alternativ kann aber auch eine Spiralklinge durch das schlitzförmige dynamische Loch eingebracht werden, welche durch eine spezielle Abschlusskappe winkelstabil verblockt wird (Abb.3,4). Diese Option ist das Standardverfahren für den PHN, in beiden Fällen ist dann die Insertion nur antegrad möglich.

Die Nagelspitze des UHN kann mit maximal drei Bolzen in zwei Ebenen in Frei-
Hand-Technik verriegelt werden. Beim PHN erfolgt diese Verriegelung maximal doppelt und parallel über den Zielbügel.

\section{Operative Techniken}

Technik der retrograden Verriegelungsnagelung mit dem UHN und generelle Besonderheiten der Oberarmmarknagelung.

Der retrograde Zugang zum Humerus bietet ausreichende und sichere Bedingungen für die Nagelung von Humerusschaftfrakturen. Ohne das Ellenbogengelenk zu eröffnen, ist dies bei Frakturen bis etwa $5 \mathrm{~cm}$ distal an die Fossa olecrani heranreichend gut möglich. Darüber hinaus können auch Frakturen im proximalen Schaftdrittel stabilisiert werden. Um hier gute Ergebnisse zu erzielen, ist allerdings auch eine präzise Vorbereitung Voraussetzung.

Bezüglich der Lagerung des Patienten sollte man sich im Klaren darüber sein, dass gerade während des Lagerungsprozesses das Risiko der zusätzlichen Weichteilschädigung und insbesondere der Schädigung des N. radialis besteht. Deswegen empfehlen wir die provisorische Oberarmschienung bis zur definitiven Lagerung des Patienten zu belassen.

\section{Lagerung}

Der Patient wird in unterpolsterte Bauchlagerung gebracht. Er liegt nahe der ipsilateralen Tischkante mit dem Kopf zur Gegenseite gedreht. Der frakturierte Oberarm wird auf einem röntgendurchlässigen Seittisch oder einem speziellen Armbrett gelagert, welches am Tisch fixiert ist. An dessen Kante wird das Ellenbogengelenk $90^{\circ}$ gebeugt, darüber hinaus 
muss das Gelenk frei für eine weitere Beugung bis $120^{\circ}$ sein.

Anschließend werden die Zugangsmöglichkeiten des Bildwandlers geprüft. Der gesamte Humerus, einschließlich Humeruskopf und Ellenbogengelenk, muss in zwei Ebenen gut darstellbar sein. Rückenoder Seitlagerung bleiben für den retrograden Zugang nur Ausnahmefällen vorbehalten.

\section{Auswahl des geeigneten Nagels}

Da der UHN in drei verschiedenen Durchmessern angeboten wird - 6,7 mm/ $7,5 \mathrm{~mm} / 9,5 \mathrm{~mm}$ - und in verschiedenen Längen verfügbar ist, müssen beide Parameter sorgfältig bestimmt werden. Der 7,5 mm-UHN stellt den Standard dar. Die Nagelung mit dem 6,7 mm-UHN wird nur bei sehr zierlichen Patienten zum Einsatz kommen, der 9,5 mm-UHN bietet sich bei osteoporotischen Knochen mit sehr weitem endomedullärem Kanal und bei pathologischen Frakturen an.

\section{Nagelmontage}

Zwei verschiedene Nagelmontagevarianten erfordern zur Entscheidung eine chirurgische Prognose vor dem Einbringen des Nagels: Wird der vorliegende Frakturtyp ohne zusätzliche Kompression heilen können oder nicht?

Im Fall der reinen Querfraktur empfehlen wir die Verwendung eines speziell für diesen Nagel entwickelten Kompressionsgerätes (Abb. 2), um die Rotationsstabilität zu erhöhen und den Frakturspalt zu schließen. Hier muss das Kompressionsgerät direkt über den Verriegelungsbügel mit dem Nagel verbunden werden.

Wenn keine zusätzliche Kompression gewünscht wird, wie z.B. bei Spiralfrakturen oder Trümmerbrüchen, wird an Stelle des Kompressionsgerätes die einfache Verbindungsschraube mit dem Bügel und Nagel verbunden. Hierbei ist wichtig, dass der Scheitelpunkt der Nagelkrümmung vom Zielbügel wegweist.

\section{Zugangsweg}

Dorsal am distalen Oberarm erfolgt die Hautinzision in Längsrichtung bis zur Olekranonspitze ziehend. Nach Spaltung der distalen Trizepsfasern wird die dorsale Fläche des distalen Humerus dargestellt ohne das Ellenbogengelenk zu eröffnen.

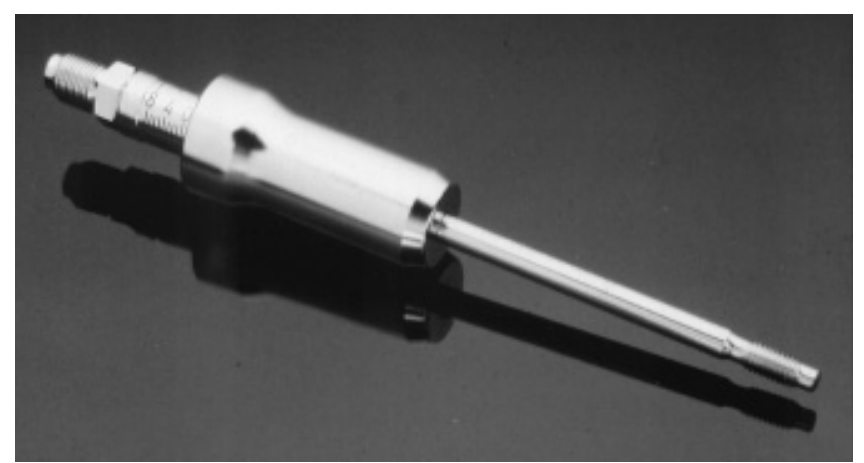

Abb. 2 Spezielles Kompressionsgerät für den UHN. Bei verriegelter Nagelspitze wird auf den dynamischen Bolzen mittels des Gerätes Druck ausgeübt. Der damit verbundene Schaftanteil wird somit zur Fraktur hin transportiert und bei dortiger Berührung weiter komprimiert. Das Ergebnis wird abschließend durch den letzten, statischen Bolzen an der Nagelbasis fixiert und das Kompressionsgerät entfernt.

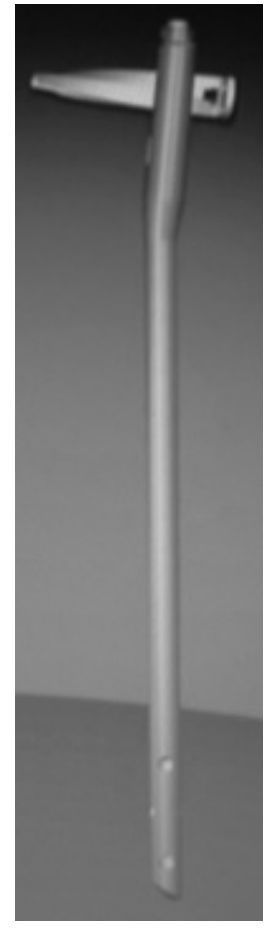

Abb. 3 Bei Kombination von diaphysären und metaphysären Humerusfrakturen wird der UHN antegrad eingebracht und, wie hier sichtbar, an seiner Basis mit dieser Spiralklinge, aber ggf. auch mit einem konventionellen zusätzlichen Bolzen, verriegelt.

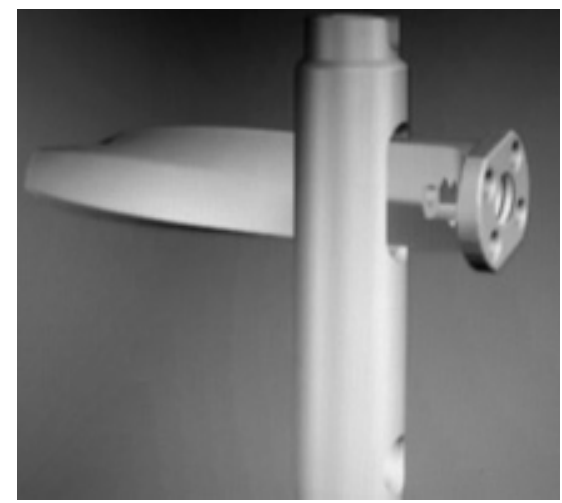

Abb. 4 Detail: Die Spiralklinge durchdringt den Nagel im dynamischen Schlitz der Nagelbasis und wird durch eine spezielle Abschlusskappe dort winkelstabil verblockt.
Der Eintrittspunkt in die Markhöhle findet sich im Zentrum eines gedachten Dreieckes zwischen der medialen und lateralen suprakondylären Kante und dem Dach der Fossa olecrani.

Drei 3,2-mm-Bohrungen senkrecht zum Markraum werden mit $4,5 \mathrm{~mm}$ überbohrt, wobei der Bohrer bis etwa $30^{\circ}$ abgesenkt wird. Der konische Fräser erzeugt eine Eintrittspforte mit $10 \mathrm{~mm}$ Breite und $20 \mathrm{~mm}$ Länge. Die distale Kante des Insertionsloches muss soweit geneigt und geglättet werden, dass der Nagel ohne Zwang eintreten kann. Bei engem Isthmus sollte man sich nicht scheuen, Handbohrer einzusetzen.

\section{Einführen des Nagels}

Der instrumentierte Nagel wird ohne Gewalt und insbesondere ohne Einschlaghammer eingebracht.

Mittels gleichmäßiger Drehbewegungen wird er zum Frakturspalt geführt und nach Reposition unter Bildwandler nur gering in die Spongiosa des Humeruskopfes inseriert, da sich im proximalen Humerusschaft noch guter Halt für die Verriegelung finden lässt. Falls man sich bei einer primären Radialisparese zur Exploration des N. radialis entscheidet, sollte dies beim Erreichen des Frakturspaltes durch den Nagel erfolgen. Hierzu eignet sich ein separater ventro-lateraler $\mathrm{Zu}$ gang in Höhe der Fraktur, in der Regel am Übergang des mittleren zum distalen Schaftdrittel.

\section{Verriegelung und interfragmentäre Kompression}

Verschiedene Verriegelungskombinationen sind sowohl proximal als auch distal 
möglich. Wir empfehlen beidseits doppelt zu verriegeln. Proximale Schaftfrakturen werden vorteilhaft proximal dreifach verriegelt.

Distal sehen wir als Standard die dorsalventrale zweifache Verriegelung durch die Zieleinrichtung des Handbügels an, allerdings sind entsprechend den verschiedenen Fraktursituationen auch hier Variationen möglich.

Wird das Kompressionsgerät eingesetzt, müssen zunächst das dynamische distale Loch und dann beide proximalen Löcher verriegelt werden. Nach erfolgter Kompression wird abschließend das distale statische Loch besetzt.

Ohne Kompressionseinheit werden initial beide distalen Löcher verriegelt, die Verwendung eines einzigen queren Bolzens bleibt Ausnahmesituationen vorbehalten.

Nach Gewährleistung einer perfekten Frakturreposition erfolgt die proximale Verriegelung in Frei-Hand-Technik mit einem röntgendurchlässigen Getriebe unter Bildwandler. Um das Innengewinde des Nagels vor Gewebswucherungen zu schützen, sollte eine Verschlusskappe verwendet werden. Verschiedene Längen dieser Verschlusskappen ermöglichen ggf. eine nachträgliche Nagelverlängerung.

\section{Technik der antegraden}

Verriegelungsnagelung mit dem UHN/PHN

- Besonderheiten im Vergleich zum retrograden Verfahren.

Das antegrade Verfahren erlaubt die Spiralklingenverriegelung, bietet sich aber auch für den konventionellen Einsatz des UHN an, wenn eine Bauchlagerung nicht zweckmäßig ist und die Beeinträchtigung des Schultergelenkes vertretbar ist bzw. durch kleine Inzisionen so gering wie möglich gehalten werden kann.

\section{Lagerung}

Zur antegraden Insertion wird der Patient halbsitzend bei $30^{\circ}$ hochgelagertem Oberkörper positioniert. Der Schulterbereich muss unter Bildwandler gut einsehbar sein. Der Arm liegt auf einer Seitenstütze.

\section{Zugang und Nagelinsertion}

Der Zugang zum Humeruskopf erfolgt über die anterolaterale Inzision vom

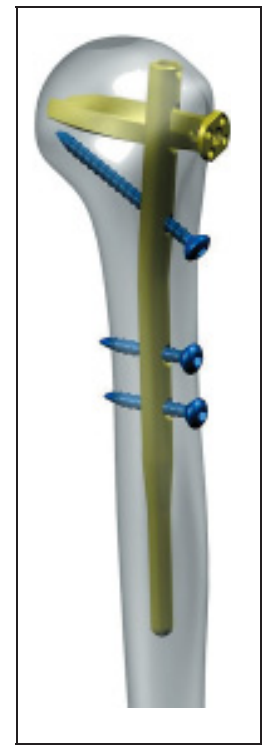

Abb. 5 Für sehr proximale Humerusschaftfrakturen ist der proximale $\mathrm{Hu}-$ merusnagel (PHN) ausreichend, welcher mit der gleichen Spiralklinge im Humeruskopf winkelstabil fixiert wird. Die distale Verriegelung erfolgt über den Zielbügel.

Akromio-Klavikular-Gelenk ausgehend, sowie die kurzstreckige Spaltung des Musculus deltoideus und der Rotatorenmanschette im Faserverlauf.

Der Insertionspunkt des Nagels befindet sich in Verlängerung der zentralen Oberarmschaftachse und etwas medial des Knochen-Knorpel-Übergang des Oberarmkopfes, um den Sehnenansatzbereich des M. supraspinatus nicht zu tangieren. Zunächst wird mit einem KirschnerDraht, dann einem durchbohrten Pfriem, in den proximalen Humerus und in den Markkanal eingegangen. Die Röntgenkontrolle in zwei Ebenen ist erforderlich.

Auswahl des Nagels, Nagelmontage und Einführen des Nagels sowie die damit verbundene Reposition der Fraktur erfolgen analog dem retrograden Verfahren.

\section{Verriegelung - Standard}

Standard ist die schräge Verriegelung, um einerseits den Humeruskopf nicht medial zu perforieren, andererseits aber medial noch guten Halt in der Gegenkortikalis zu erzielen. Aber auch die doppelt-parallele Verriegelung ist hier möglich. Wichtig ist, dass die Nagelbasis durch ihre Basiskrümmung nach lateral und nicht nach ventral zeigt. Dadurch kann distal doppelt ventrodorsal in Freihand-Technik verriegelt werden.

\section{Verriegelung - Spiralklinge}

Wird eine stabilere Verankerung im Humeruskopf mittels Spiralklinge erwünscht, wird nach Positionierung des Nagels - gleichgültig ob UHN oder
PHN - immer zuerst die proximale Spiralklingen-Verriegelung mit dem schrägen Zielbügelaufsatz durchgeführt.

Der Nagel muss hierbei so unter die Kopfoberfläche versenkt werden, dass er auch mit Verschlussschraube die Kalotte nicht überragt, aber auch die Spiralklinge selbst nicht zu tief positioniert ist. Idealerweise liegt sie am Übergang vom mittleren zum caudalen Drittel des Humeruskopfes. Nach der lateralen Hautinzision wird ein Kirschner-Draht durch eine Bohrbüchse eingebohrt und unter Bildwandler dessen definitive Lage zentral im Humeruskopf kontrolliert. Die Spiralklingenlänge wird bemessen, die Eintrittsstelle unikortikal aufgebohrt. Die Spiralklinge wird nun mittels Führung durch den Zielbügelaufsatz bis an den Nagelschlitz vorgetrieben und mit vorsichtigen Hammerschlägen in die gewünschte Position gebracht, wobei sie gerade subchondral zu platzieren ist. Hierbei macht sie eine $90^{\circ}$-Drehung und stützt den $\mathrm{Hu}-$ merus horizontal mit ihrer breiten Klingenfläche ab.

Wichtig ist der Verschluss der Nagelbasis mit einer speziellen Abschlusskappe, die ihrerseits durch Druck auf die Spiralklinge jene winkelstabil komprimiert.

Falls erforderlich, werden die Tuberkula über Zuggurtungsfäden an den Perforationen der Nagelbasis befestigt.

Die distale Verriegelung kann beim PHN dann über den Zielbügel erfolgen, beim UHN hingegen in Freihand-Technik doppelt von anterior nach posterior.

\section{Postoperative Weiterversorgung}

Gewöhnlich wird postoperativ keine zusätzliche Schienung erforderlich. Schulter- und Ellenbogenübungen können sofort gebahnt werden, allerdings sollten Rotationsbewegungen gegen Widerstand bis zur Knochenheilung unterbleiben.

Die Implantentfernung ist routinemäßig nicht erforderlich und sollte Ausnahmesituationen vorbehalten bleiben.

\section{Klinische Erfahrungen}

\section{Intraoperative Erfahrungen}

Die Einführung des UHN im Jahre 1995 wurde dadurch erleichtert, dass bei den Autoren zuvor Erfahrungen bei Humerusschaftfrakturen einerseits mit der antegraden und retrograden Verriegelungs- 


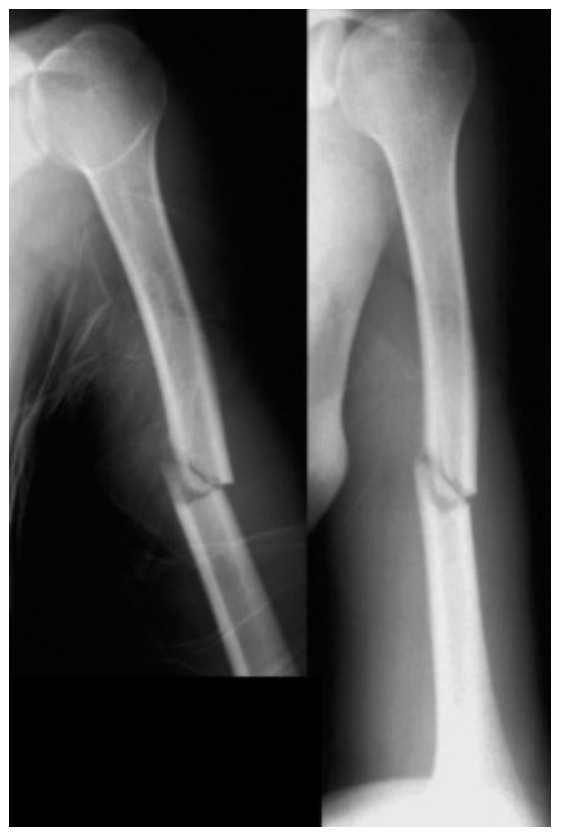

C

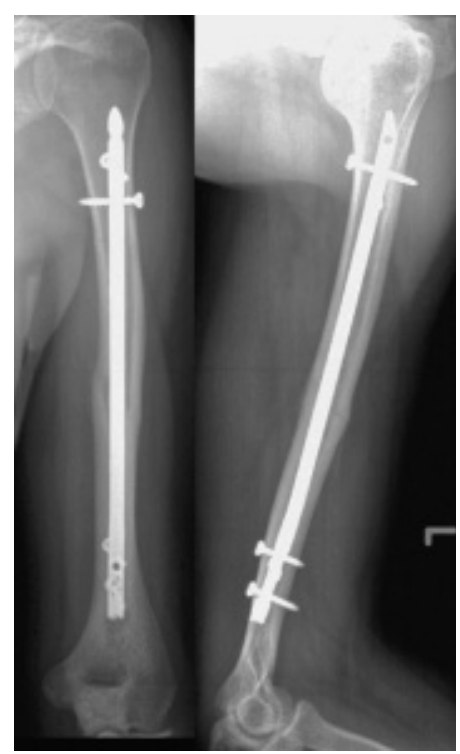

nagelung mittels Russel-Taylor-Nagel, andererseits mit der retrograden Bündelnagelung bestanden.

Insbesondere die Unzufriedenheit mit den technischen Unzulänglichkeiten des Instrumentariums und Designs dieser intramedullären Implantate floss in die Entwicklung des UHN ein. Dies spiegelt sich in einem recht unkomplizierten Handling dieses Implantates in der eigenen Klink wider. Die Verwendung der Titanlegierung anstelle von Stahl und die damit verbundene höhere Elastizität erleichtern insbesondere die Insertion des Nagels beim retrograden Vorgehen und mindert die Gefahr der zusätzlichen Fissur oder Fraktur im kondylären Bereich.

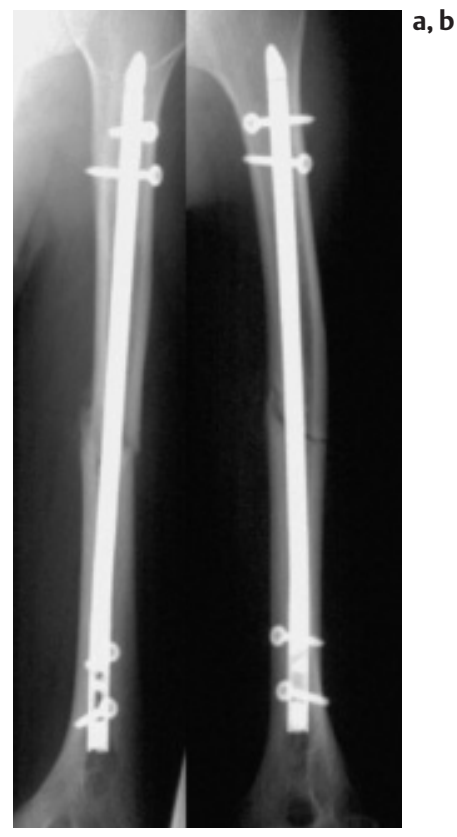

Abb. 6 a Kurze Schrägfraktur des Humerus, a.p. und seitlich, $\mathbf{b}$ retrograde Insertion eines 7,5-mm-UHN, interfragmentäre Kompression, statische Verriegelung, postoperativ, a.p. und seitlich, c knöcherne Konsolidation nach 13 Wochen, a.p. und seitlich.

Auch trägt das Design des Zielbügels zu diesem Ziel bei, da er symmetrisch und ohne Erhöhung des vom Nagel vorgegebenen Durchmessers gestaltet ist. Dadurch werden die Hebelkräfte am Insertionsloch minimiert. Beim antegraden Verfahren bleibt die Kompromittierung der Weichteile durch den Zielbügel ebenfalls verringert.

Sicherlich gestaltet sich die Einführung des Humerusnagels für Chirurgen, die sich bis dahin primär mit der Plattenosteosynthese bei Humeruschaftfrakturen befasst haben, zunächst schwieriger, als für Chirurgen mit einer langjährigen
Nagelungspraxis. Dies zeigen die Ergebnisse einer ersten Multizenterstudie mit dem UHN.

Bei der Dokumentierung der intraoperativen Probleme standen vor allem für die mit der intramedullären Behandlung von Humerusfrakturen wenig vertrauten Chirurgen Schwierigkeiten im Vordergrund, die im Sinne einer „Learning curve“ mit steigenden Fallzahlen gut beherrschbar waren. Dies betraf beim retrograden Verfahren (Abb. 6 u. 7) die erschwerte Verriegelung in Freihand-Technik an der proximalen Nagelspitze (5,8\%), Fissurenbildung oder Aussprengungen an der Nageleintrittsstelle (3,9\%), schwierige Nagelinsertion (2,9\%), zusätzliche Handaufbohrung $(2,0 \%)$, insuffiziente Bolzenstabilität, Unstimmigkeit in der Nagellängenmessung und zusätzliche Schaftfraktur (je 1\%). Bei 3,9\% Patienten wurden sekundäre Paresen des N. radialis festgestellt. Hier lagen Traktionsschäden vor, die sich insbesondere durch artgerechte Lagerung in der Regel vermeiden lassen. So sollte der bei Aufnahme angelegte Oberarmgips erst nach definitiver Bauchlagerung entfernt werden und beim Hautdesinfizieren darauf geachtet werden, dass der betroffene Oberarm nicht in der Frakturregion „durchhängt“ oder torquiert wird (Tab.1).

Beim antegraden Vorgehen (Abb. 8) ergaben sich mit der Insertion keine wesentlichen Schwierigkeiten, allerdings wurde in 3,7\% der Fälle ein geringer Halt eines Bolzens, sowie in 7,4\% Schwierigkeiten beim Verriegeln der Nagelspitze in Freihandtechnik berichtet.

Sinnvoll ist sicherlich, für die Verriegelung der Nagelspitze nur eine Hautinzision durchzuführen, die darunter liegende Muskulatur bis auf den Schaft zu spreizen, aber auch den Bolzen beim

Tab. 1 Klinische Erfahrungen intraoperativ

\begin{tabular}{lr} 
Intraoperative Probleme & \\
\hline UHN - retrogrades Vorgehen & $5,8 \%$ \\
erschwerte Verriegelung in Freihand-Technik an der Nagelspitze & $3,9 \%$ \\
Fissurenbildung oder Aussprengungen an der Nageleintrittsstelle & $2,0 \%$ \\
schwierige Nagelinsertion (2,9\%), zusätzliche Handaufbohrung & $1,0 \%$ \\
insuffiziente Bolzenstabilität & $1,0 \%$ \\
Unstimmigkeit in der Nagellängenmessung & $1,0 \%$ \\
zusätzliche Schaftfraktur & \\
UHN - antegrades Vorgehen & $3,7 \%$ \\
geringer Halt eines Bolzens & $7,4 \%$ \\
Schwierigkeiten beim Verriegeln der Nagelspitze in Freihand-Technik & $3,9 \%$ \\
sekundäre Paresen des N. radialis &
\end{tabular}


a, b
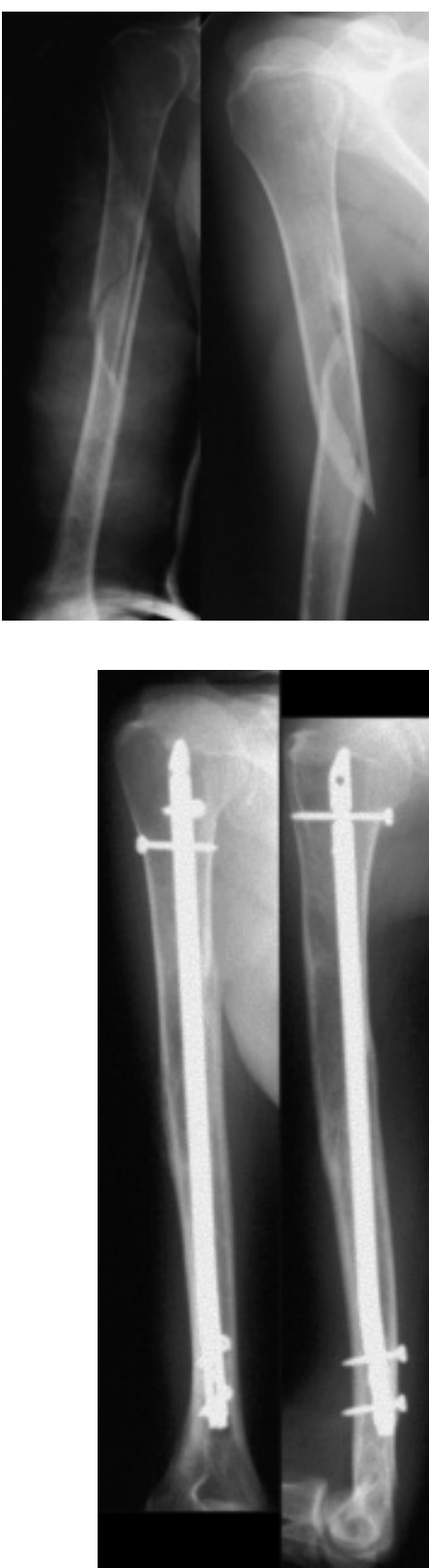

Einschrauben mit einem Faden zu sichern, um ihn bei einem Verlust in den Weichteilen problemlos bergen zu können [4].

In 4 Fällen mussten zusätzliche Implantate zur verbesserten Frakturstabilität eingebracht werden: in einem Fall wurden bei Primäroperation zusätzliche Schrauben, einmal Zerklage-Draht und einmal zusätzliche Platten und Schrauben verwendet. In einem weiteren Fall mussten zusätzliche Zugschrauben in der ersten Woche nach Nagelung ergänzt werden.

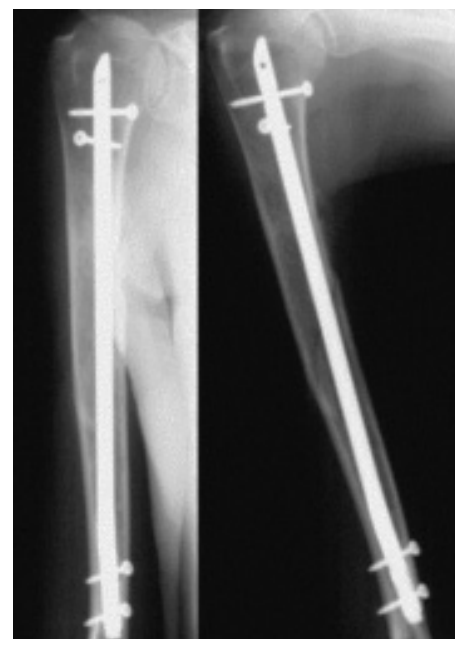

Abb. 7 a Spiralfraktur des Humerus nach Bagatelltrauma bei Patienten mit langjähriger Kortisontherapie, auffallend weite Markhöhle, a. p. und seitlich, $\mathbf{b}$ retrograde Insertion eines 9,5-mm-UHN, statische Verriegelung, postoperativ, a. p. und seitlich, c knöcherne Konsolidation nach 16 Wochen, a.p. und seitlich.

Die Schnitt-Naht-Zeit lag sowohl beim antegraden als auch beim retrograden Verfahren in zwei Drittel der Fälle unter 60 Minuten.

Die Erfahrung bezüglich des intraoperativen Handlings mit der Spiralklinge beim UHN (Abb.9), wie auch mit dem PHN (Abb.10), ist allerdings noch deutlich geringer, da diese erst ab Oktober 2000 bzw. Juni 2001 verfügbar waren. Bis jetzt wurden 52 Frakturen mit diesen Implantaten behandelt. Prinzipiell ähneln sich die intraoperativen Erfahrungen im Umgang mit beiden Implantaten. Unterschiedlich ist im Wesentlichen der distale Verriegelungsmodus, der sich beim PHN sehr einfach und zeitsparend erweist, da dies über den Zielbügel geführt erfolgt. Hier ergaben sich weder Fehlverriegelungen noch spätere Bolzenlockerungen. Die distale Verriegelung des antegrad eingebrachten UHN ist von der Versorgung der Schaftfrakturen bekannt und geübt. Die Freihand-Verriegelung ist zeitintensiver und erfordert nach einer Stichinzision der Haut die stumpfe Dissektion der ventral gelegenen Muskulatur und vorsichtige Präparation auf den Schaft, um Gefäßoder Nervenläsionen auszuschließen. Auch hier ergaben sich weder Fehlverriegelungen, spätere Bolzenlockerungen, noch Verletzungen von Nerven oder Gefäßen.
Zur winkelstabilen Verblockung der Spiralklinge im Nagel muss eine Abschlusskappe in die Basis eingeschraubt werden. Diese stand initial nur als $5 \mathrm{~mm}$ verlängernde Abschlusskappe zur Verfügung, welche im Gegensatz zur 0-mm-Abschlusskappe nicht über den Hohlraum des Zielbügels eingebracht werden kann. In drei von 52 Fällen wurde während dieses Verschlussaktes die Kappe in den Weichteilen bzw. in der Humeruskopfspongiosa verloren und musste mühsam geborgen werden. Die Sicherung der Kappe mit einem Vicrylfaden erleichterte dieses Problem, mit der 0-mmAbschlusskappe ergab es sich erst gar nicht.

\section{Postoperative Erfahrungen}

75 Patienten mit retrograder Nagelung $[2,18,20]$ konnten die prospektiv bis zur Knochenheilung weiter verfolgt werden. 58 dieser 75 komplett dokumentierten Fälle besaßen frische Frakturen, 9 Pseudarthrosen, 6 pathologische Frakturen und 2 Refrakturen. Von den nichtpathologischen Frakturen gehörten 40 zum ATyp, 23 zum B-Typ und 6 zum C-Typ.

Die iatrogenen Paresen des N. radialis erholten sich ohne weitere Eingriffe. Knochen- oder Wundinfekte waren nicht zu verzeichnen.

$52 \%$ der Frakturen heilten innerhalb von drei Monaten nach Nagelung aus, weitere 37,3\% innerhalb der ersten 6 Monate. 4\% der Frakturen benötigten bis zu acht Monate. Bei 5 Patienten (6,7\%) mit Knochenheilungsproblemen wurden in einem Fall eine Spongiosatransplantation und eine neue distale Verriegelung durchgeführt. In drei Fällen wurde das Kompressionsgerät benutzt, wobei in einem Fall ein neuer Nagel und in einem Fall eine zusätzliche Spongiosatransplantation notwendig wurden. Im fünften Fall wurde eine Plattenosteosynthese ausgeführt (Tab. 2).

In vier Fällen wurden Implantate direkt nach Knochenheilung entfernt: in drei Fällen das komplette Nagelsystem und in einem Fall lediglich die Verriegelungsschrauben.

Nach Frakturheilung beklagten drei von 75 Patienten (4\%) Schulterschmerzen, zwei Patienten $(2,7 \%)$ berichteten von bedeutsamen Schmerzen der Ellenbogenregion. 

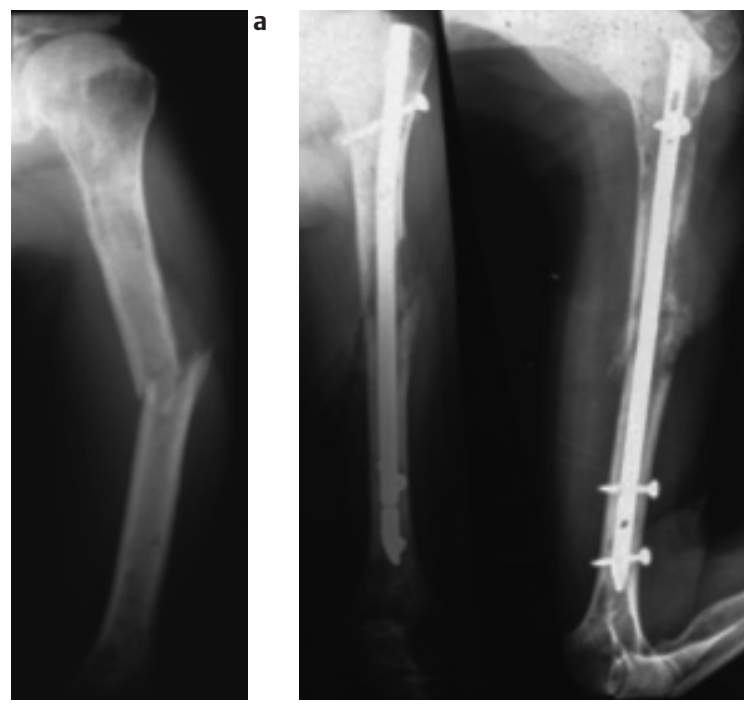

b Abb. 8 a Pathologische Fraktur des Humerusschafts bei Metastasierung aufgrund eines Mammakarzinomes, a.p., b antegrade Insertion eines 7,5-mm-UHN, proximale schräge statische Verriegelung, a.p. und seitlich, $c$ Situation nach 6 Monaten, a.p. und seitlich.

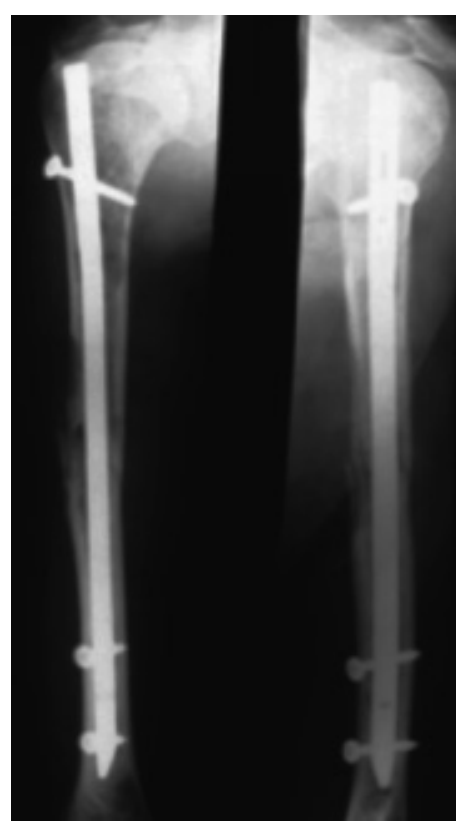

c

eine befriedigende und 1,8\% eine schlechte Schulterfunktion, wobei $87,4 \%$ eine exzellente, $10,8 \%$ eine befriedigende und 1,8\% eine schlechte Ellenbogenfunktion aufwiesen.

Bei der antegraden Insertion [4] ergab sich ein sehr gutes Bewegungsergebnis für das Ellenbogengelenk und eine im Vergleich zum Ellenbogengelenk etwas weniger gute Bewertung bei der Schulterbeweglichkeit.

Die Chirurgen der Multizenterstudie selbst bewerteten den Verlauf abschließend in 90,7\%, die Patienten in 94,7\% der Fälle als exzellent oder gut.

Bei dem UHN mit Spiralklinge bzw. PHN ergab sich in 5 von 52 Fällen durch die 5mm-Abschlusskappe ein relativer Nagelhochstand, welcher allerdings nur in einem Fall eine wirkliche Impingementsymptomatik provozierte. In vier Fällen erfolgte die Metallentfernung nach Konsolidierung der Fraktur. Auch das Problem des Nagelüberstandes konnte mit der Verwendung der 0-mm-Abschlusskappe gelöst werden, die somit als Standard weitere Verwendung fand [6].

In einem Fall musste eine Standard-UHNAbschlusskappe durch eine Spiralklingenabschlusskappe ausgetauscht werden, da die Klinge nicht winkelstabil verblockt war. Bei den mit dem UHN und Spiralklinge behandelten Humerus-

Zur Beurteilung der Schulter- und Ellenbogenfunktion wurde nach Knochenheilung das Bewegungsausmaß von Schulterabduktion/adduktion, Schulterextension/flexion, Schulterexorotation/endorotation, Ellenbogenextension/flexion und Ellenbogenpronation/supination sowohl des frakturierten als auch des gesunden Armes aufgezeichnet. Die Schulter- und Ellenbogenfunktion wurde, wenn weniger als $10^{\circ}$ Verlust des Bewegungsausmaßes in jeder Richtung bestanden, als exzellent, bei Verlust des Bewegungsausmaßes zwischen $10^{\circ}$ und $30^{\circ}$ als befriedigend und bei Verlust des Bewegungsausmaßes von mehr als $30^{\circ}$ als schlecht eingestuft.

Bei der retrograden Insertion hatten 89,2\% der Patienten eine exzellente, 9\%

Tab. 2 Klinische Erfahrungen postoperativ

\begin{tabular}{lr}
\hline Frakturheilung & \\
\hline Frakturheilung innerhalb von drei Monaten nach Nagelung & $52,0 \%$ \\
Frakturheilung zwischen 3 und 6 Monaten & $37,3 \%$ \\
Frakturheilung zwischen 6 und 8 Monaten & $4,0 \%$ \\
Knochenheilungsprobleme & $6,7 \%$ \\
$\quad$ Lösung & 1 \\
Spongiosatransplantation und neue distale Verriegelung & 1 \\
Plattenosteosynthese & 3 \\
interfragmentäre Kompressionsgeräte & 1 \\
$\quad$ darunter neue Nagelung & 1
\end{tabular}

Beweglichkeit nach Ausheilung

UHN - retrogrades Vorgehen

Schulterfunktion exzellent

befriedigend schlecht

Ellenbogenfunktion exzellent

$87,4 \%$

befriedigend schlecht

UHN - antegrades Vorgehen

Schulterfunktion exzellent

befriedigend

$11,1 \%$ schlecht

Ellenbogenfunktion exzellent

befriedigend schlecht 


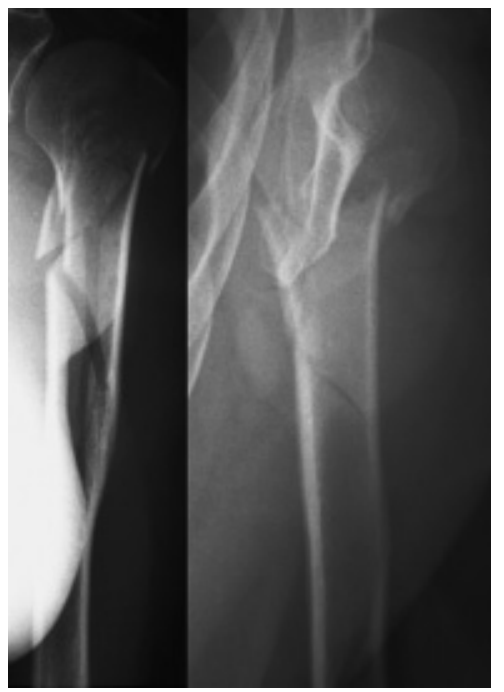

a
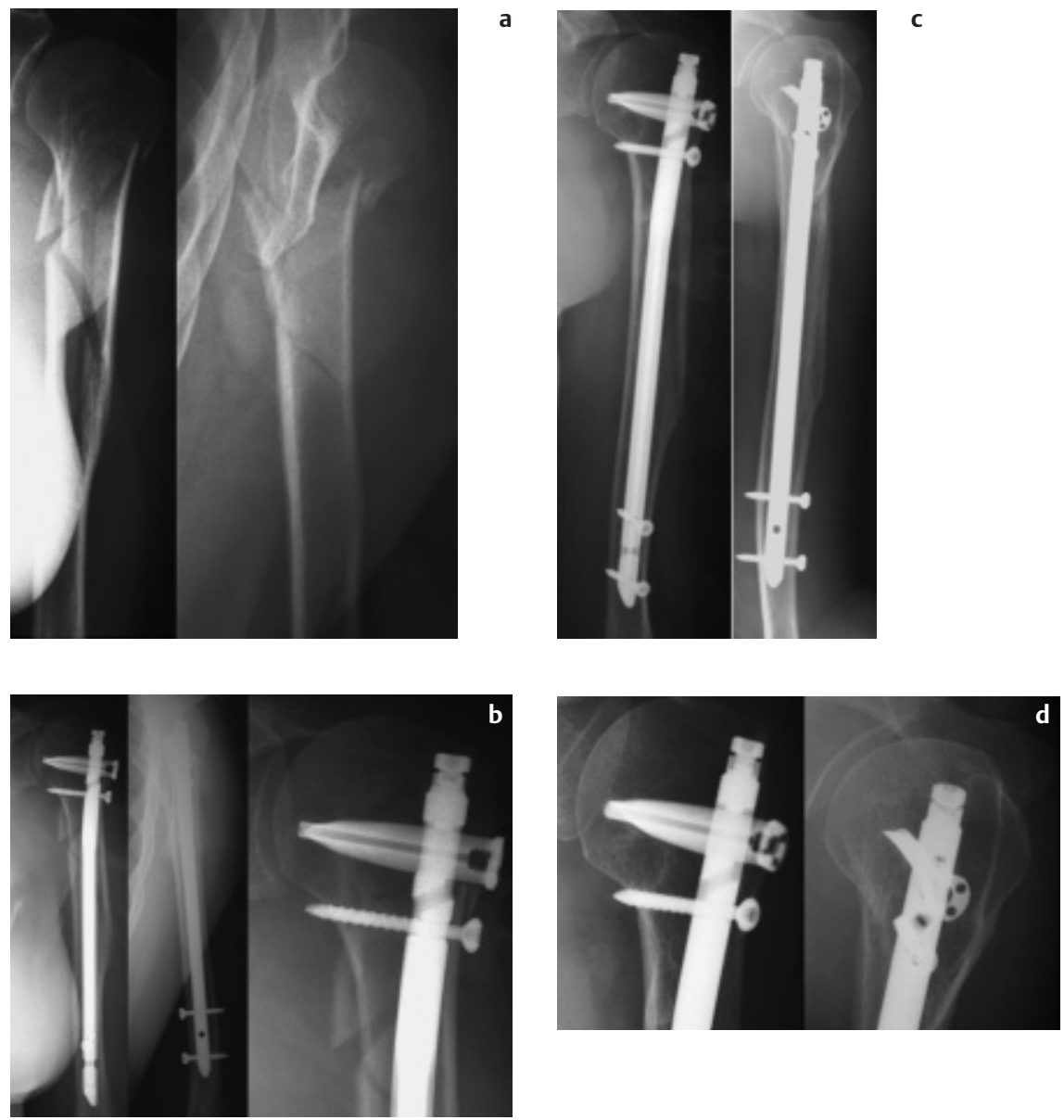

Abb.9 a Humerusschaftfraktur von der Schaftmitte nach proximal reichend, b geschlossene Stabilisierung mit antegradem 7,5-mm-UHN und Spiralklinge, a.p. und seitlich, sowie Detail vom Humeruskopf, c knöcherne Konsolidierung, Röntgenkontrolle nach 5,5 Monaten, d Detail vom Humeruskopf.

schaftfrakturen ergaben sich bisher keine wesentlichen Heilungsstörungen. In einem Fall mit großem metaphysären Knochendefekt wurde durch Implantation eines UHN mit Spiralklinge bewusst geschlossen reponiert und stabilisiert, eine lateral betonte Defektzone zunächst akzeptiert, aber bereits primär eine sekundäre Spongiosaplastik geplant. Nach Spongiosaplastik kam es innerhalb von 4 Monaten zur Konsolidierung der Fraktur. Für die definitive Beurteilung klinischer Ergebnisse von Humerusschaftfrakturen mit metaphysärer Komponente (UHN mit Spiralklinge) und sehr proximal gelegene Schaftfrakturen (PHN) sind die Fallzahlen noch zu klein und der Nachbeobachtungszeitraum zu kurz.

\section{Diskussion}

Die Verriegelungsmarknagelung bei Schaftfrakturen langer Röhrenknochen ist ein international weit verbreitetes und akzeptiertes Verfahren. Aus den ers- ten Entwicklungen von Marknägeln durch Gerhart Küntscher sind ganze Familien ausgereifter Marknagelsysteme entstanden, die qualitativ sehr hoch stehend und verlässlich anzusehen sind. Für die diaphysären Frakturen von Tibia und Femur muss man dieses Verfahren inzwischen als Standard ansehen. Aber auch für den Humerus hat sich in den letzten 6 Jahren ein deutlicher Trend hin zur Marknagelung gezeigt [2,4,16, $17,20]$. Dies hängt mit dem verbesserten Design moderner Humerusmarknägel und deren Instrumentarium zusammen, welche eine sichere und stabile Versorgung der Oberarmschaftfrakturen ermöglichen. Aber auch die Senkung der Invasivität und Gelenkbelastung dieser modernen Implantate hat hier einen positiven Beitrag geleistet. Hinzu kommt die zunehmende Vertrautheit von Unfallchirurgen mit diesen Systemen, welche das Risiko zusätzlicher iatrogener Verletzungen während der Implantation senken konnte.
Wie bei anderen Implantatsystemen auch, fällt und steigt die Erfolgsrate auch bei der Verwendung des UHN mit der Präzision der Implantation und der Beachtung deren technischer Details. Die Indikationsstellung muss abgesichert, Implantat und Instrumentarium müssen in allen Größen verfügbar und in funktionablem Zustand sein.

Ein zu dicker Marknagel kann insbesondere den distalen Schaftanteil sprengen, ein zu langes Implantat entweder überstehen oder perforieren.

Die Verwendung grober Kraft beim Einbringen des Marknagels bei retrograder Insertion kann zu zusätzlichen Frakturen oder Fissuren der kondylären Region des Humerus führen.

Hier darf nur mit Handkraft gearbeitet werden und gegebenenfalls muss das Insertionsloch erweitert geformt werden.

Die Bauchlagerung des Patienten erfolgt sinnvollerweise noch mit anliegendem Oberarmgips, um das Risiko von Traktionsschäden des $\mathrm{N}$. radialis zu senken. Dies gilt auch für die antegrade Insertion des Nagels, bei der die distale Verriegelung nicht von lateral, sondern von ventral erfolgt. Generell sollte die Verriegelung nur über kurze Hautinzisionen, und dann stumpfe Präparation mit Spreizung der Muskulatur auf den Schaft erfolgen, um Nerven- oder Gefäßschäden generell zu vermeiden.

Spezifische Fraktursituationen erfordern unter Umständen auch den Einsatz weiterer Implantate. Für eine ungestörte Knochenheilung ist eine gute Fragmentadaptation notwendig. Dies gilt insbesondere für die langstreckige Spiralfraktur mit weit abstehenden Frakturspitzen, bei denen ggf. eine Drahtzerklage sinnvoll ergänzt. Bestehen zusätzliche metaoder gar epiphysäre Frakturanteile, so können beispielsweise Ethibondzuggurtungen oder Schraubenosteosynthesen notwendig werden [6].

Bei stark osteoporotisch geschwächtem Humerus kann auch bei reinen Schaftfrakturen der antegrade Nagel sinnvoll mit der Spiralklinge kombiniert werden, da hier die abstützende Potenz dieser winkelstabilen proximalen Verriegelung im Vergleich zur reinen Bolzenverriegelung höher ist.

Der Einsatz interfragmentärer Kompression sollte bei Quer- und kurzen Schrägfrakturen bedacht werden, da diese bei 

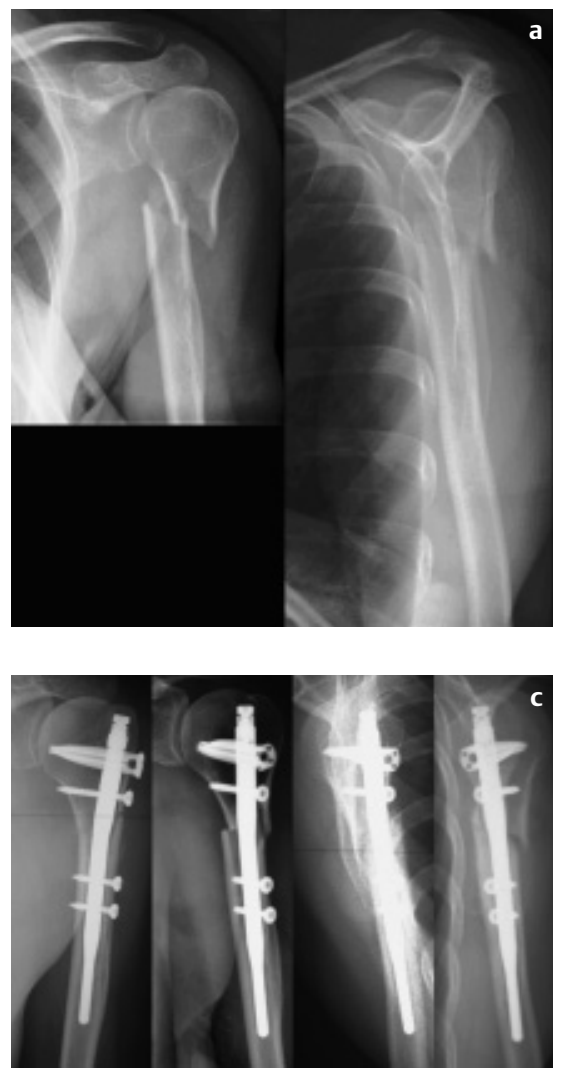

Abb.10 a Sehr proximal gelegene Humerusschaftfraktur, b intraoperativer Situs unter dem Bildwandler bei geschlossener Stabilisierung mit dem proximalen Humerusnagel (PHN), mit dem hier eine distale Verriegelung über den Zielbügel unterhalb der Fraktur möglich ist, c postoperative Situation bei Röntgenkontrolle in 4 Ebenen nach 3 Wochen, $\mathbf{d}$ postoperative Situation nach 6 Monaten mit knöcherner Konsolidierung der Fraktur.
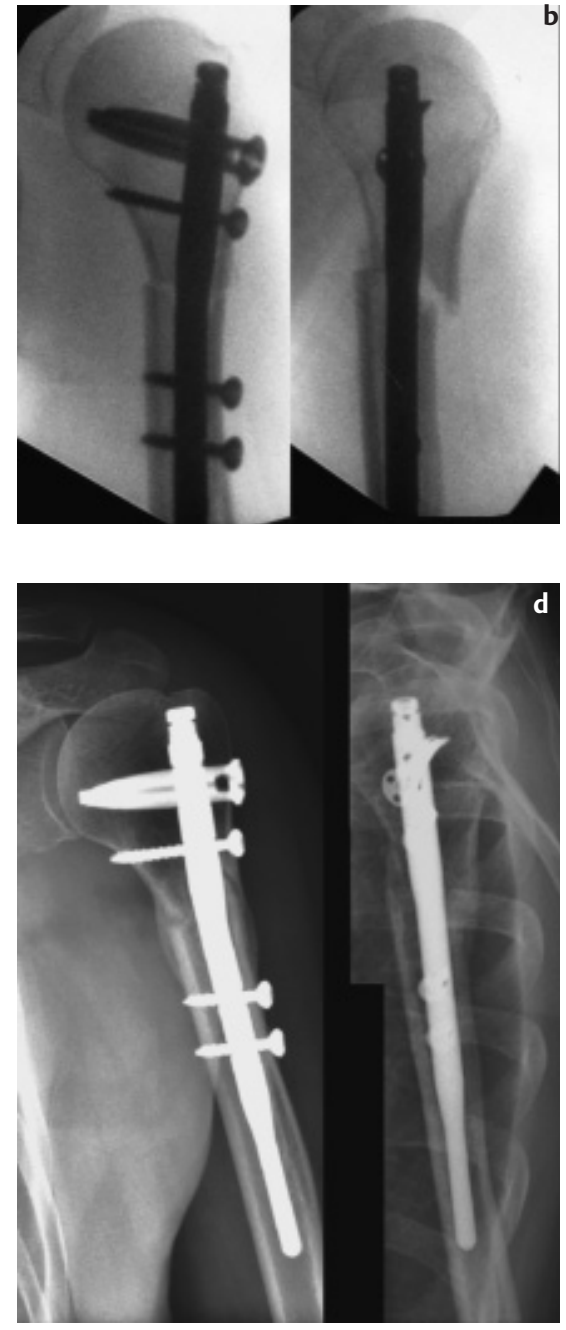

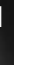
steosynthese mit dem Nagel bedacht werden (Abb.8).

Wir sehen auch bei der Humerusschaftpseudarthrose durchaus eine Indikation für den UHN. Allerdings muss von Fall zu Fall sehr kritisch abgewogen werden, ob nicht neben der intrafragmentären Kompression auch aufgebohrt werden und/oder die Knochentransplantation erfolgen sollte.

\section{Fazit}

Zusammenfassend beurteilen wir den unaufgebohrten Humerusnagel (UHN) als ein ausgereiftes Implantat in der Vervollständigung der unaufgebohrten Verriegelungsnägel für Femur und Tibia an. Er wird seiner Aufgabe gerecht, bei schonendem Zugangsweg und biologischer Behandlung der Frakturzone eine Frakturstabilisierung mit einem hohen Maß
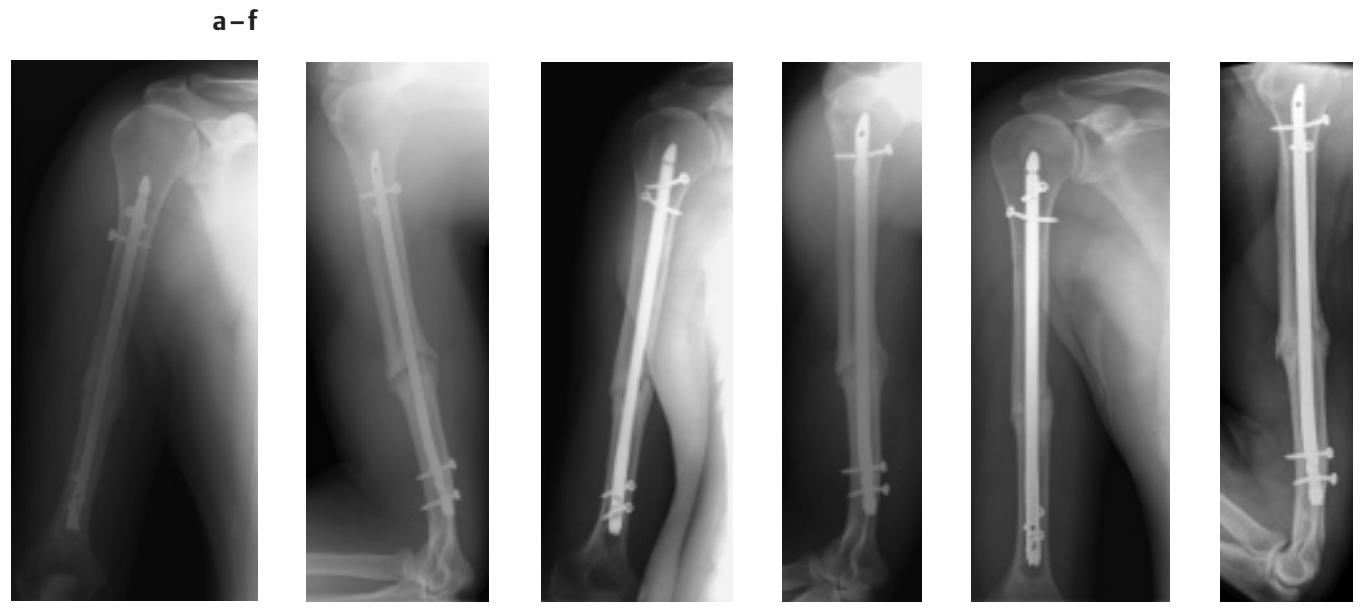

Abb.11 a + b Zugewiesener Patient mit Pseudarthrose des Humerusschaftes nach unkomprimierten Marknagelung mit UHN 7,5 mm Durchmesser und $260 \mathrm{~mm}$ Länge. Röntgenkontrolle präoperativ in 2 Ebenen, c $+\mathbf{d}$ Entfernung des ungebohrten Humerusmarknagels, Aufbohren der Markhöhle, Implantation eines UHN 9,5 mm Durchmesser und $280 \mathrm{~mm}$ Länge, proximal und distal je doppelt verriegelt. Interfragmentäre Kompression mit gewünschter Bolzenverklemmung. Situation bei Röntgenkontrolle direkt postoperativ in 2 Ebenen, $\mathbf{e}+\mathbf{f}$ Ausheilung der Pseudarthrose nach 4 Monaten, Röntgenkontrolle in 2 Ebenen. 
an Rotationsstabilität zu gewährleisten. Die guten Ergebnisse der Schulter- und Ellenbogengelenksbeweglichkeit, insbesondere beim retrograden Verfahren, teilweise schon in den ersten postoperativen Wochen, verstärken diese positiven Erfahrungen.

Auf dieser Grundlage propagieren wir den retrograden Zugang als Standardzugang für die operative Behandlung von reinen Humerusschaftfrakturen. Der antegrade Zugang kann bei kurzer Inzision ebenfalls mit geringer Morbidität angewandt werden. Wir setzen ihn vor allem bei bereits reduzierter Beweglichkeit im Schultergelenk, oder in spezifischer Fraktursituation ein, die eine Bauchlagerung erschweren. Auch ist er der geeignete Zugang, wenn die Verriegelungsoption einer Spiralklinge erwünscht wird. Dies betrifft vor allem die Kombination einer diaphysären und metaphysären Fraktur des gleichen Oberarmes, aber auch die Implantation des PHN bei sehr proximal gelegenen Schaftfrakturen.

Neben der akuten Oberarmschaftfraktur lässt sich die Verriegelungsmarknagelung auch bei pathologischen Frakturen, Refrakturen und atrophen wie hypertrophen Pseudarthrosen gewinnbringend einsetzen (Abb.11).

\section{Literatur}

${ }^{1}$ Bell MJ, Beauchamp CG, Kellam JK, McMurtry RY. The results of plating humeral shaft fractures in patients with multiple injuries: the Sunnybrook experience. J Bone Joint Surg (Br) 1985; 67-B: 293-296

${ }^{2}$ Blum J, Rommens PM, Janzing H, Langendorff HS. Retrograde Nagelung von Humerusschaftfrakturen mit dem UHN - eine internationale multizentrische Studie. Unfallchirurg 1998; 101: $342-352$

${ }^{3}$ Blum J, Rommens PM. Interfragmentäre Kompression bei der Humerusschaftnagelung mit einem speziellen Kompressionsgerät. Akt Traumatol 2001; 31: 90 -94

${ }^{4}$ Blum J, Janzing H, Gahr R, Langendorff HS, Rommens PM. Clinical performance of a new medullary humeral nail: antegrade versus retrograde insertion. J Orthop Trauma 2001; 15: 342-349

${ }^{5}$ Blum J, Rommens PM. Proximale Verriegelung von Humerusmarknägeln und Verletzungsrisiko des $\mathrm{N}$. axillaris. Unfallchirurg 2002; 105: 9-13

${ }^{6}$ Blum J, Hessmann MH, Rommens PM. Behandlung proximaler metaphysärer Humerusfrakturen mit Verriegelungsmarknagelung und Spiralklinge - erste Erfahrungen mit einem neuen Implantat. Akt Traumatol 2003; 33: im Druck

${ }^{7}$ Böhler L. Gegen die operative Behandlung von frischen Oberarmschaftbrüchen. Langenbecks Arch Klin Chir 1964; 308: $465-$ 475

${ }^{8}$ Brug E, Westphal T, Schäfers G. Differenzierte Behandlung der Humerusdiaphysenfrakturen. Unfallchirurg 1994; 97: 633-638

${ }^{9}$ Ikpeme JO. Intramedullary interlocking nailing for humeral fractures: experiences with the Russell-Taylor humeral nail. Injury 1994; 25: $447-55$

${ }^{10}$ Kelsch G, Deffner P, Ulrich C. Die Humerusverriegelungsnagelung nach Seidel - Klinische Ergebnisse nach 100 Anwendungen. Unfallchirurg 1997; 100: 111 -118

${ }^{11}$ Nast-Kolb D, Knoefel WT, Schweiberer L. Die Behandlung der Oberarmschaftfraktur. Ergebnisse einer prospektiven AO-Sammelstudie. Unfallchirurg 1991; 94: 447-454

${ }^{12}$ Riemer BL, Butterfield SL, D’Ambrosia R, Kellam J. Seidel intramedullary nailing of humeral diaphyseal fractures: a preliminary report. Orthopedics 1991; 14: 239-246

${ }^{13}$ Riemer BL, Foglesong ME, Burke CJ, Butterfield SL. Complications of Seidel intramedullary nailing of narrow diameter humeral diaphyseal fractures. Orthopedics 1994; 17: $19-29$.
${ }^{14}$ Robinson CM, Bell KM, Court-Brown CM, McQueen MM. Locked nailing of humeral shaft fractures: experience in Edinburgh over a two-year period. J. Bone Joint Surg (Br) 1992; 74-B: 558-562

${ }^{15}$ Rommens PM, Vansteenkiste F, Stappaerts $\mathrm{KH}$, Broos PL. Indikationen, Gefahren und Ergebnisse der operativen Behandlung von Oberarmfrakturen. Unfallchirurg 1989; 92 : 565-570

${ }^{16}$ Rommens PM, Verbruggen J, Broos PL. Retrograde Verriegelungsnagelung der Humerusschaftfraktur. Unfallchirurg 1995; 98: $133-$ 138

${ }^{17}$ Rommens PM, Verbruggen J, Broos PL. Retrograde locked nailing of humeral shaft fractures. A review of 39 patients. J. Bone Joint Surg 1995; 77-Br: 84-89.

${ }^{18}$ Rommens PM, Blum J, Runkel M. Retrograde nailing of humeral shaft fractures. Clin. Orthop. 1998; 350: 26-39

${ }^{19}$ Rommens PM, Endrizi DP, Blum J, White RR. Humerus - Shaft. In: Rüedi T, Murphy WM. (Hrsg). AO Principles of Fracture Management. Stuttgart: Thieme, 2000: 291-305

${ }^{20}$ Rommens PM, Blum J. Die retrograde Nagelung mit dem UHN - Klinische Ergebnisse. Akt Traumatol 2001; 31: 125-132

${ }^{21}$ Seidel H. Humeral locking nail: a preliminary report. Orthopedics 1989; 12: 219-226

${ }^{22}$ Siebert CH, Heinz BC, Höfler HR, Hansis M. Plattenosteosynthetische Versorgung von Humerusschaftfrakturen. Unfallchirurg 1996; 99: 106-111

${ }^{23}$ Varley GW. The Seidel locking humeral nail: the Nottingham experience. Injury 1995; 26 : $155-157$

Priv.-Doz. Dr. med. Jochen Blum Oberarzt

Dr. med. Erik Hanke

Assistenzarzt

Dr. med. Philip Höhle

Assistenzarzt

Prof. Dr. med. Pol M. Rommens

Direktor

Klinik und Poliklinik für

Unfallchirurgie der

Johannes Gutenberg Universität Mainz Langenbeckstr. 1

D-55131 Mainz 